\title{
Association of TM6SF2 rs58542926 with Hepatic and Extrahepatic Alterations in Chronic Hepatitis C Patients
}

Mohi I. Mohammed Abdul

Taibah University

Adriana Vitiello

Padua University: Universita degli Studi di Padova

Hanieh Ghassabian

University of Padova: Universita degli Studi di Padova

Heba M. Eltahir

Taibah University

Elham Ahmed Hassan

Assiut University Faculty of Medicine

Amany A. Bekhit

Minia University

Abo Bakr F. Ahmed

Minia University

Hany E.A. Ahmed

Taibah University

Meky M. Abouzied

Minia University

Hossein Elbadawy ( $\nabla$ hmbadawy@taibahu.edu.sa )

Taibah University https://orcid.org/0000-0001-8675-0838

Arianna Calistri

University of Padova: Universita degli Studi di Padova

Gualtiero Alvisi

Padua University: Universita degli Studi di Padova

Khaled Thabet

Minia University

\section{Research Article}

Keywords: TM6SF2, rs58542926, chronic hepatitis C, thrombocytopenia 
Posted Date: August 17th, 2021

DOl: https://doi.org/10.21203/rs.3.rs-803234/v1

License: (c) (i) This work is licensed under a Creative Commons Attribution 4.0 International License. Read Full License 


\section{Abstract}

Human genetic variants play major roles in predicting and prognosis of several diseases. The effect of rs58542926 variant in transmembrane 6 superfamily member 2 (TM6SF2) gene on liver fibrosis among patients with chronic hepatitis $\mathrm{C}(\mathrm{CHC})$ is still debatable. The aim of this study is to investigate the possible effects of this variant in $\mathrm{CHC}$ patients. The study comprised 351 subjects: $250 \mathrm{CHC}$ patients with different fibrosis stages (F0-F4) and 101 healthy volunteers. TM6SF2 (rs58542926) genotype was determined for all subjects. Blood samples were collected for complete blood count and biochemical analysis and cohort subjects were genotyped for the variant TM6SF2 rs58542926. Fibrosis staging was performed using Fibrotest and Fibroscan standard tests. The presence of the minor allele was significantly associated with severe liver fibrosis, as well as thrombocytopenia as an extrahepatic alteration. In addition, there was a significant association between the minor allele and lower thrombopoietin levels. The association of TM6SF2 genotype with thrombocytopenia was explored by measuring plasma thrombopoietin (TPO) levels for $\mathrm{CHC}$ patients. The results showed an association with extrahepatic alteration (thrombocytopenia) through its effect on plasma TPO level, and consequently on platelets production, which raises questions about the role of this variant in HCV treatment outcome. In conclusion, the occurrence of the minor allele of the variant rs 58542926 can be linked to severe fibrosis stages as well as thrombocytopenia, enabling this variant to be used as a diagnostic pharmacogenetic marker for predicting the risk of fibrosis onset in $\mathrm{CHC}$ patients.

\section{Introduction}

Hepatitis $C$ virus is a pervasive virus affecting millions around the world, where Egypt shows one of the highest prevalence ratios of about $14 \%$ and about 40,000 deaths per year. Besides being a worldwide health hazard, the burden of the disease is still far from being controlled. A large percentage of the patients $(70-80 \%)$ fails to get rid of the virus spontaneously during the acute phase and develop chronic infections ${ }^{1}$. Chronic Hepatitis $\mathrm{C}(\mathrm{CHC})$ viral infection is accompanied by both hepatic- and extrahepatic complications so that mortality rates doubles in HCV carriers, as compared to healthy individuals ${ }^{2}$. Liver cirrhosis and hepatocellular carcinoma are the worst-expected hepatic complications of $\mathrm{CHC}$ infections. However, the highest mortality rates from HCV infections has been correlated to progressive hepatic fibrosis ${ }^{3}$. Fibrosis is a structural damage to the liver tissue that ranges from mild changes limited to the portal and peripheral areas, to more severe changes that end up with cirrhosis in a time frame of approximately 20 years. Fibrosis is believed to be a result of many etiologies other than $\mathrm{CHC}$, including obesity, alcohol intake and chronic viral infections. These multiple etiological factors suggest that genetic factors may also plays a pivotal role in the pathogenesis of chronic liver disease 4 .

Even with recent advances in the eradication of HCV using direct-acting antivirals (DAAs), a limited population of patients with genetic variances seems to be resistant to the treatment, or more susceptible to liver fibrosis secondary to $\mathrm{CHC}$ infection ${ }^{5,6}$. The transmembrane 6 superfamily 2 (TM6SF2) variant is located to locus 19p13.3-p12, and was identified by an exome-wide association study ${ }^{7}$. The TM6SF2 
rs58542926 non-synonymous polymorphism was associated with non-alcoholic fatty liver disease (NAFLD) ${ }^{7,8}$, higher prevalence of fibrosis and alcohol-related cirrhosis ${ }^{9}$, triglyceride secretion ${ }^{10}$ and myocardial infarction ${ }^{11}$. This variant was also shown to result in fat accumulation in the liver and reduce the very low density lipoprotein ( $V L D L)$ secretion in vitro ${ }^{7}$. Therefore, the association between the singlenucleotide polymorphism (SNP) rs58542926 in the TM6SF2 gene was hypothesized to play a role in liver fibrosis in $\mathrm{CHC}$ patients. At the beginning, the role of rs 58542926 variant was debatable in case of $\mathrm{CHC}$, until an Australian large scale functional study showed a significant correlation of this variant with liver fibrosis in $\mathrm{CHC}^{12}$. This study was followed by a meta-analysis which supported the role of this variant in histological changes in $\mathrm{CHC}$ infection, where it showed a robust association between the TM6SF2 variant and fibrosis development ${ }^{13}$. This study, therefore, aims at investigating the role of rs 58542926 variant and its association with both hepatic and extrahepatic alteration in Egyptians $\mathrm{CHC}$ patients.

\section{Patients And Methods}

\section{Patient cohort}

For gaining representative results, the study included 351 subjects ( 250 with $\mathrm{CHC}$, and 101 healthy control subjects) from Assiut university hospital, Assiut, Egypt. The inclusion criteria taken in consideration for selecting $\mathrm{CHC}$ subjects included $\mathrm{CHC}$ patients who had a fibroscan and fibrotest ${ }^{\circledR}$ with scoring for fibrosis stage before initiating the antiviral treatment. Patients who had evidence of other liver diseases by standard tests were excluded. An informed consent was obtained from all patients who agrees to be included in this study. The healthy control group included individuals with no history of chronic liver diseases. All experiments were performed in compliance with relevant laws and institutional guidelines and in accordance with the ethical standards of the Declaration of Helsinki. Ethical approval was obtained from the research ethics committee in the Faculty of Pharmacy, Minia University (No. HV01/2020) before the commencement of this work. Each subject included in the study signed a written informed consent, and was informed regarding the nature of the disease and the diagnostic procedures involved

\section{Clinical and laboratory assessment}

Samples and data were collected at the time of liver fibrosis staging assessment. viral load, serum bilirubin, serum albumin, Aspartate transaminase (AST), alanine transaminase (ALT), international normalized ratio (INR) and platelets count were evaluated using commercially available kits.

\section{Staging of liver fibrosis}

Both FibroScan ${ }^{\circledR}$, a non-invasive test for liver fibrosis assessment, and FibroTest ${ }^{\circledR}$, a highly sensitive serum biomarker test for evaluating liver fibrosis, were used for staging fibrosis to avoid the use of invasive techniques like taking biopsies ${ }^{11}$. Fibrosis was staged according to Metavir scoring system. Fibrosis was scored on a 5-point scale: stage zero (F0): no fibrosis; stage one (F1): portal fibrosis alone; 
stage two (F2): portal fibrosis with rare septa; stage three (F3): portal fibrosis with many septa; and stage four (F4): cirrhosis. A total of 73 patients out of 250 patients were categorized as F0-F1, 50 patients were categorized as F2, 26 patients as F3, while the remaining 91 patients were categorized as F4.

\section{Genotyping}

Genotyping for TM6SF2 rs58542926 ( $n=351)$ was performed using the TaqMan SNP genotyping allelic discrimination method according to manufacturer's instructions (Applied Biosystems, Foster City, CA). All genotyping was blinded to clinical variables.

\section{Determination of TPO level}

Thrombopoietin level was measured by a solid phase sandwich enzyme-linked immunosorbent assays (ELISA) (Invitrogen, USA) in plasma of CHC patients with known TM6SF2 rs58542926 genotype.

\section{Statistical analysis}

Statistical analyses of the data were carried out using GraphPad Prism version 8.0 (Graph pad software San Diego, USA). Difference in the median values were measured using the two-tailed Mann-Whitney ttest. $P$ values less than 0.05 were considered statistically significant.

\section{Results}

\section{Association between the rs 58542926 genotype and clinical, viral, and metabolic characteristics in CHC.}

Comparing the frequency of the minor allele frequency (MAF) of TM6SF2 rs58542926 in healthy group and $\mathrm{CHC}$ subjects showed almost similar values in both groups, where the minor allele represented about $10 \%$ in healthy group and $9 \%$ in $\mathrm{CHC}$ group $(\mathrm{MAF}=0.06)(P=0.7$ for trend) $($ Table 1$)$.

Table 1

\begin{tabular}{|lll|}
\hline Distribution of TM6SF2 rs58542926 and Hardy-Weinberg equilibrium \\
\hline TM6SF2 rs58542926 & $\begin{array}{l}\text { CHC } \\
\text { (Egyptian patients) } \\
(\mathbf{n}=\mathbf{2 5 0 )}\end{array}$ & $\begin{array}{l}\text { Healthy controls } \\
\text { (Egyptian population) } \\
(\mathbf{n}=101)\end{array}$ \\
\hline CC & $230(92 \%)$ & $91(90 \%)$ \\
CT & $18(7 \%)$ & $9(9 \%)$ \\
\hline TT & $2(1 \%)$ & $1(1 \%)$ \\
\hline
\end{tabular}

Table 1 showing $p=0.7$ for both $\mathrm{CHC}$ and healthy controls cohorts. $\mathrm{P}$ values were calculated by chi square test, $p>0.05$ indicates no deviation from Hardy-Weinberg equilibrium 
When the distribution of the major and minor alleles in males and females of each group was compared, it was found that the percent of females carrying the minor alleles was higher, however, the difference was statistically non-significant $(p=0.893)$. Patients with the TM6SF2 rs58542926 (TT/CT) genotype had no significant difference in HCV viral load compared to subjects with CC genotype, despite the lower mean value for viral load in the former group. When comparing CT/TT versus CC regarding the metabolic profile, subjects with minor allele had significantly higher serum bilirubin, higher INR ratios and lower platelet count. It is to be noted that no significant differences were observed between the minor allele group (CT/TT) and the major allele one regarding the mean values of ALT, AST, serum albumin and BMI, as seen in table 2.

Table (2) Characteristics of Egyptian CHC patients according to TM6SF2 rs58542926 Genotype.

\begin{tabular}{|llll|}
\hline \multirow{2}{*}{ Variables } & \multicolumn{2}{l}{ TM6SF2 rs58542926 Genotype } & \multirow{2}{*}{ P value } \\
\cline { 2 - 3 } & $\mathbf{C C}$ & $\mathbf{C T / T T}$ & \\
& $(\mathbf{n}=\mathbf{2 3 0})$ & $\mathbf{( n = 2 0 )}$ & \\
\hline Age & $55.6 \pm 13.6$ & $56.1 \pm 12.2$ & 0.893 \\
\hline Gender (M/F) \% & $132 / 98(57.4 / 42.6 \%)$ & $11 / 9(55 / 45 \%)$ & 0.836 \\
\hline BMI (Kg/m $\left.{ }^{2}\right)$ & $27.9 \pm 6.2$ & $28.6 \pm 5.9$ & 0.783 \\
\hline Serum bilirubin & $0.8(0.2-3.9)$ & $1.1(0.4-6.95)$ & $0.014^{*}$ \\
\hline Serum albumin & $3.6 \pm 1$ & $3.1 \pm 1$ & 0.068 \\
\hline Platelet (x10 $/ \mathrm{L})$ & $190.3 \pm 91.9$ & $135.7 \pm 73.2$ & $0.01^{*}$ \\
\hline ALT (IU/L) & $37(3-259)$ & $34(13-146)$ & 0.375 \\
\hline AST (IU/L) & $40(9-320)$ & $44.5(19-191)$ & 0.274 \\
\hline INR & $1.2 \pm 0.4$ & $1.5 \pm 0.5$ & $0.008^{*}$ \\
\hline HCV viral load & $8.8 \times 10^{5}$ & $6.4 \times 10^{5}$ & 0.328 \\
\hline
\end{tabular}

\section{Association between TM6SF2 variant and plasma thrombopoietin level in CHC patients}

After observing the significant association of the minor allele with thrombocytopenia, it was interesting to explore whether this low platelet count can be regarded to a decrease in thrombopoietin levels, the primary regulator of platelet production. Measuring plasma thrombopoietin level for $31 \mathrm{CHC}$ patients showed a significant association between rs58542926 minor allele and low plasma thrombopoietin level as shown in Fig. $1(p<0.05)$. 


\section{Association between TM6SF2 variant and fibrosis stage in CHC patients}

After evaluation of the fibrosis stage with Fibrotest and Fibroscan, we next analyzed the association between TM6SF2 rs58542926 genotype and liver fibrosis stage. Interestingly, The rs 58542926 minor allele showed a significant association with severe fibrosis stages $(70 \%$ of subjects had staging of $\mathrm{F} 3$ or more) compared to the major allele where $65 \%$ of subjects showed fibrosis stages of F0-F2 at most. ( $P=$ $0.005)$ as shown in Fig. 2.

\section{Discussion}

The current study explored the effect of the TM6SF2 E167K variant on liver fibrosis severity in Egyptian patients with $\mathrm{CHC}$. Based on our findings, it is evident that the TM6SF2 E167K variant favors the development of both hepatic and extrahepatic alterations. The TM6SF2 E167K minor allele was associated with severe liver fibrosis stages as reported before ${ }^{13}$, but we also found an association with thrombocytopenia in an Egyptian cohort of 250 patients with $\mathrm{CHC}$ viral infection. Previous studies, however, analyzed the contribution of TM6SF2 E167K variant to the pathogenicity of nonalcoholic steatohepatitis ${ }^{14-16}$.

The most important hepatic alterations which occur due to $\mathrm{CHC}$ infection are liver cirrhosis, hepatocellular carcinoma (HCC) and end-stage liver disease, where the progression from fibrosis to cirrhosis can take ten to thirty years ${ }^{17}$.

In addition, $\mathrm{CHC}$ patients with chronic $\mathrm{HCV}$ infection also suffer from several extra-hepatic pathologies that range from stroke and myocardial infarction to diabetes and autoimmune responses like rheumatoid arthritis. Thrombocytopenia is a major concern in $\mathrm{CHC}$ patients, affection about $45 \%$ of them. This decrease in platelet count can induce bleeding manifestations, which strongly can influence the initiation and continuation of antiviral therapy in the corresponding cases. Thrombocytopenia can be observed in the majority of HCV patients with advanced fibrosis and/or cirrhosis, compared with the non-cirrhotic patients ${ }^{18,19}$. Thrombocytopenia can limit not only diagnostic but also therapeutic procedures and treatments, and increases risk of complications, especially excessive bleeding ${ }^{20}$.

Several etiologies have been proposed for the pathogenesis of thrombocytopenia in $\mathrm{CHC}$ patients including excessive destruction of platelets either via autoimmune reactions or platelet sequestration as a result of splenomegaly ${ }^{21-23}$. In addition, two further mechanisms are also possible as a cause for $\mathrm{CHC}$ associated thrombocytopenia, including virus-induced bone marrow suppression and decreased TPO production ${ }^{20}$. There are also data supporting the coexistence of several mechanisms causing thrombocytopenia in response to chronic HCV and cirrhosis ${ }^{21}$. Therefore, more research is needed to 
clarify the underlying factors behind the pathogenesis of chronic liver diseases-associated thrombocytopenia.

Human genetic variants are gaining much interest in the last decade for their role in chronic liver diseaseseverity and its-associated thrombocytopenia. Interestingly, a previous study showed a significant association between the minor allele of rs11697186 (located in DDRGK1 gene) and the decrease in platelet count that was observed during the treatment of HCV infection with peg-interferon ${ }^{24}$.

In the current study, we found a significant association between the minor allele ( $T$ allele) and the occurrence of thrombocytopenia which agrees with previous research reporting this extrahepatic manifestation in $\mathrm{CHC}$ patients $4,18,21,25$. It is worth to note that the observed thrombocytopenia in the current study was linked to lower TPO plasma levels these findings are in agreement with data from previous studies which have detected a negative correlation between the production of TPO and the degree of liver fibrosis, one possible explanation for the association between the TM6SF2 minor allele and low plasma TPO level is the association of this allele with advanced-stage liver fibrosis. As fibrosis advances to cirrhosis, liver shrinks and loses its ability to synthesize TPO, resulting in inappropriately low levels of TPO ${ }^{26}$.

\section{Conclusion}

In conclusion, our study shed the light on the association of the minor allele of rs 58542926 and serum TPO levels with the fibrosis stage in Egyptian CHC patients, and links the minor allele to the severe fibrosis stages in such cohort. This introduces the minor variant of TM6SF2 rs58542926 as a pharmacogenetic diagnostic tool for predicting fibrosis progression in $\mathrm{CHC}$ patients.

\section{Declarations}

\section{Acknowledgements}

This work was supported financially by a Strategic grant number 13-MED615-05 provided by the National Science, Technology and Innovation Plan (NSTIP) from King Abdulaziz City for Science and Technology (KACST) as part of the Strategic Technologies Programs of the National Plan for Science, Technology and Innovation (MAARIFAH).

\section{Conflict of interests}

The authors declares no conflict of interests

\section{References}

1. Lavanchy D (2009) The global burden of hepatitis C. Liver international 29:74-81 
2. El-Kamary SS, Jhaveri R, Shardell MD (2011) All-cause, liver-related, and non-liver-related mortality among HCV-infected individuals in the general US population. Clinical infectious diseases 53(2):150-157

3. Thrift AP, El-Serag HB, Kanwal F (2017) Global epidemiology and burden of HCV infection and HCVrelated disease. Nature reviews Gastroenterology hepatology 14(2):122

4. Chen X, Zhou P, De L, Li B, Su S (2019) The roles of transmembrane 6 superfamily member 2 rs58542926 polymorphism in chronic liver disease: A meta-analysis of 24,147 subjects. Molecular genetics genomic medicine 7(8):e824

5. Noureddin M, Wright EC, Alter HJ et al (2013) Association of IL28B genotype with fibrosis progression and clinical outcomes in patients with chronic hepatitis $\mathrm{C}$ : a longitudinal analysis. Hepatology 58(5):1548-1557

6. McKiernan SM, Hagan R, Curry M et al (2004) Distinct MHC class I and II alleles are associated with hepatitis C viral clearance, originating from a single source. Hepatology 40(1):108-114

7. Kozlitina J, Smagris E, Stender S et al (2014) Exome-wide association study identifies a TM6SF2 variant that confers susceptibility to nonalcoholic fatty liver disease. Nature genetics 46(4):352

8. Liu Y-L, Reeves HL, Burt AD et al (2014) TM6SF2 rs58542926 influences hepatic fibrosis progression in patients with non-alcoholic fatty liver disease. Nature communications 5:4309

9. Buch S, Stickel F, Trépo E et al (2015) A genome-wide association study confirms PNPLA3 and identifies TM6SF2 and MBOAT7 as risk loci for alcohol-related cirrhosis. Nature genetics 47(12):1443

10. Mahdessian H, Taxiarchis A, Popov S et al. TM6SF2 is a regulator of liver fat metabolism influencing triglyceride secretion and hepatic lipid droplet content. Proceedings of the National Academy of Sciences. 2014;111(24):8913-8918

11. Holmen OL, Zhang H, Fan Y et al (2014) Systematic evaluation of coding variation identifies a candidate causal variant in TM6SF2 influencing total cholesterol and myocardial infarction risk. Nature genetics 46(4):345

12. Eslam M, Mangia A, Berg T et al (2016) Diverse impacts of the rs58542926 E167K variant in TM6SF2 on viral and metabolic liver disease phenotypes. Hepatology 64(1):34-46

13. Liu Z, Que S, Zhou L et al (2017) The effect of the TM6SF2 E167K variant on liver steatosis and fibrosis in patients with chronic hepatitis C: a meta-analysis. Scientific reports 7(1):1-11

14. Dongiovanni P, Petta S, Maglio C et al (2015) Transmembrane 6 superfamily member 2 gene variant disentangles nonalcoholic steatohepatitis from cardiovascular disease. Hepatology 61(2):506-514

15. Luukkonen PK, Zhou Y, Haridas PN et al (2017) Impaired hepatic lipid synthesis from polyunsaturated fatty acids in TM6SF2 E167K variant carriers with NAFLD. Journal of Hepatology 67(1):128-136

16. Chen L-Z, Xia HH-X, Xin Y-N, Lin Z-H, Xuan S-Y (2015) TM6SF2 E167K variant, a novel genetic susceptibility variant, contributing to nonalcoholic fatty liver disease. Journal of clinical translational hepatology 3(4):265 
17. Poynard T, Bedossa P, Opolon P (1997) Natural history of liver fibrosis progression in patients with chronic hepatitis C. The Lancet 349(9055):825-832

18. Giannini E (2006) Thrombocytopenia in chronic liver disease and pharmacologic treatment options. Alimentary pharmacology therapeutics 23(8):1055-1065

19. Louie K, Micallef J, Pimenta J, Forssen U (2011) Prevalence of thrombocytopenia among patients with chronic hepatitis C: a systematic review. Journal of viral hepatitis 18(1):1-7

20. Rawi S, Wu GY (2020) Pathogenesis of thrombocytopenia in chronic HCV infection: a review. Journal of Clinical Translational Hepatology 8(2):184

21. Tejima $\mathrm{K}$, Masuzaki $\mathrm{R}$, Ikeda $\mathrm{H}$ et al (2010) Thrombocytopenia is more severe in patients with advanced chronic hepatitis $C$ than $B$ with the same grade of liver stiffness and splenomegaly. Journal of gastroenterology 45(8):876-884

22. Zucker M, Hagedorn C, Murphy C, Stanley S, Reid K, Skikne B (2012) Mechanism of thrombocytopenia in chronic hepatitis $\mathrm{C}$ as evaluated by the immature platelet fraction. International journal of laboratory hematology 34(5):525-532

23. Hernández F, Blanquer A, Linares M, López A, Tarín F, Cerveró A (1998) Autoimmune thrombocytopenia associated with hepatitis $C$ virus infection. Acta haematologica 99(4):217-220

24. Tanaka Y, Kurosaki M, Nishida N et al (2011) Genome-wide association study identified ITPA/DDRGK1 variants reflecting thrombocytopenia in pegylated interferon and ribavirin therapy for chronic hepatitis C. Human molecular genetics 20(17):3507-3516

25. Tampaki M, Koskinas J (2014) Extrahepatic immune related manifestations in chronic hepatitis $\mathrm{C}$ virus infection. World Journal of Gastroenterology: WJG 20(35):12372

26. Adinolfi LE, Giordano MG, Andreana A et al (2001) Hepatic fibrosis plays a central role in the pathogenesis of thrombocytopenia in patients with chronic viral hepatitis. British journal of haematology 113(3):590-595

\section{Figures}

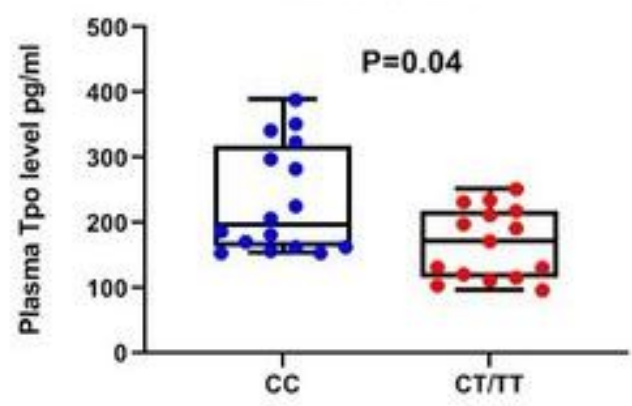

Figure 1 
Association of rs58542926 genotype with plasma thrombopoietin level in patients with $\mathrm{CHC}(\mathrm{n}=33)$. Pvalues are univariate and provided for the dominant model of inheritance

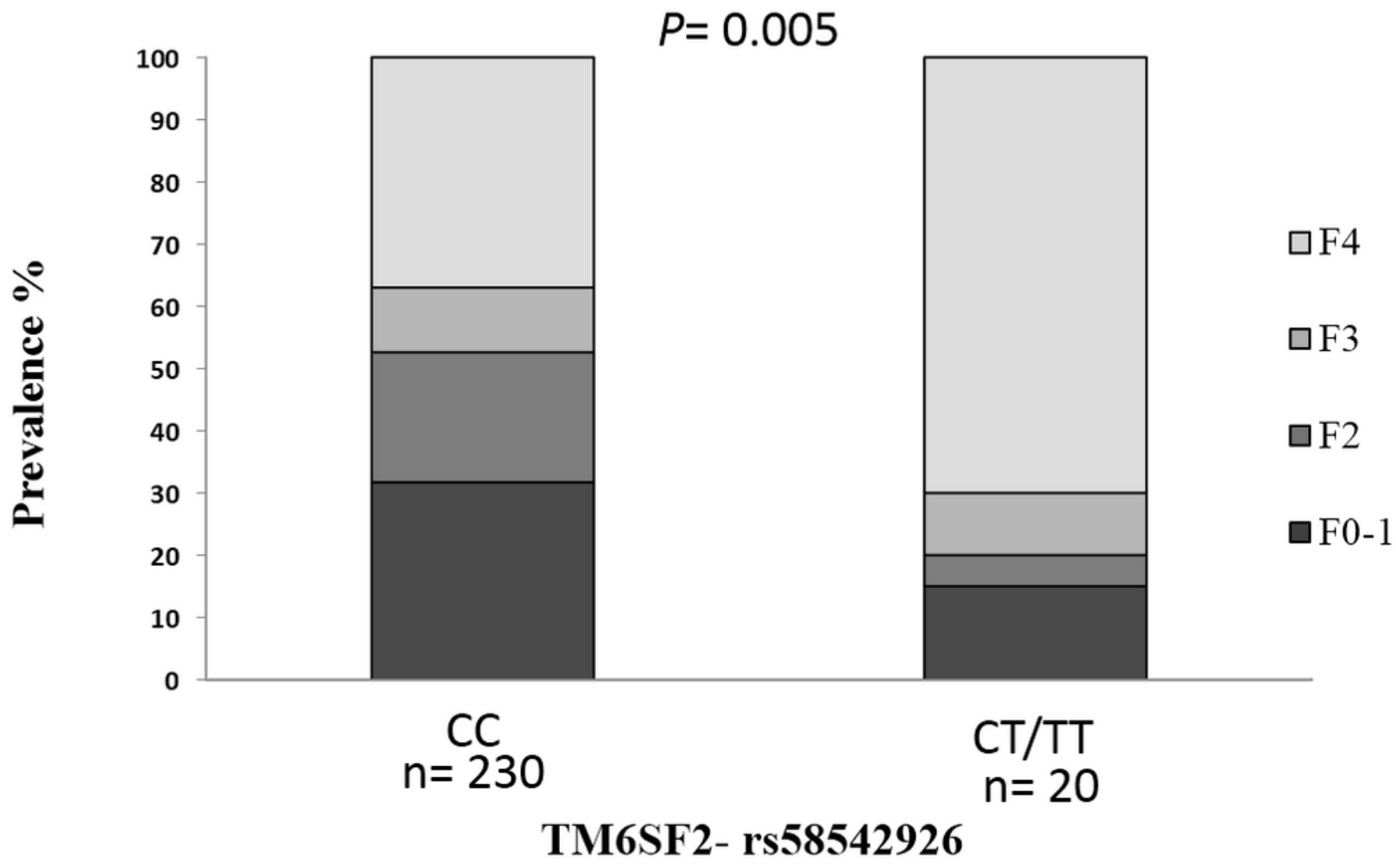

Figure 2

Association of rs58542926 genotype with fibrosis stage in the Egyptian cohort $(n=250)$. P-values are univariate and provided for the dominant model of inheritance 